\title{
The On/Off nature of star-planet interactions in the HD 179949 and $v$ And systems
}

\author{
Evgenya Shkolnik, ${ }^{1}$ David A. Bohlender, ${ }^{2}$ Gordon A. H. Walker ${ }^{3}$ and \\ Andrew Collier Cameron ${ }^{4}$ \\ ${ }^{1}$ NASA Astrobiology Institute / University of Hawaii \\ 2680 Woodlawn Drive, Honolulu, HI 96822 \\ email: shkolnik@ifa.hawaii.edu \\ ${ }^{2}$ Herzberg Institute for Astrophysics, National Research Council of Canada \\ Victoria BC, Canada V9E 2E7 \\ ${ }^{3} 1234$ Hewlett Place, Victoria, BC, V8S 4P7 \\ ${ }^{4}$ School of Physics and Astronomy, University of St. Andrews, North Haugh, St. Andrews, \\ Fife, Scotland KY16 9SS
}

\begin{abstract}
Evidence suggesting an observable magnetic interaction between a star and its hot Jupiter $\left(P_{\text {orb }}<7\right.$ days, $\left.a<0.1 \mathrm{AU}, M_{p} \sin i>0.2 \mathrm{M}_{J}\right)$ appears as a cyclic variation of stellar activity synchronized to the planet's orbit. HD 179949 has been observed almost every year since 2001. Synchronicity of the Ca II H \& K emission with the orbit is clearly seen in four out of six epochs, while rotational modulation with $P_{r o t}=7$ days is apparent in the other two seasons. We observe a similar phenomenon on $v$ And, which displays rotational modulation $\left(P_{\text {rot }}=12\right.$ days $)$ in September 2005, while in 2002 and 2003 variations appear to correlate with the planet's orbital period. This on/off nature of star-planet interaction (SPI) in the two systems is likely a function of the changing stellar magnetic field structure throughout its activity cycle. The tentative correlation between this activity in the 13 stars we have observed to date and the ratio of $M_{p} \sin i$ to the planet's rotation period, a quantity proportional to the hot Jupiter's magnetic moment, first presented in Shkolnik et al. (2005) remains viable. This work furthers the characterization of SPI, improving its potential as a probe of extrasolar planetary magnetic fields.
\end{abstract}

Keywords. late-type stars, chromospheric activity, planetary systems, HD 179949, $v$ And

\section{Introduction}

Observations and theory demonstrate that star-planet interaction (SPI) is a complex, yet potentially very informative probe of extrasolar planetary magnetic fields. In Shkolnik et al. (2003, 2005), we reported on planet-induced chromospheric activity on two stars, HD 179949 and $v$ And apparent from the night-to-night modulation of the Ca II H \& K chromospheric emission phased with the hot Jupiter's orbit. The modulation indicated of a magnetic rather than tidal interaction (Cuntz et al. 2000), such that the period of the observed stellar activity correlated with the planet's orbital period $P_{\text {orb }}$, rather than $P_{\text {orb }} / 2$.

One scenario of magnetospheric interaction proposes that the planet induces reconnection and flaring events as it travels through the large stellar magnetic loops (Cuntz et al. 2000, Saar et al. 2005, Ip et al. 2004), implying that the resulting activity should 
depend on the star's magnetic field, the planet's magnetic field and the orbital distance with respect to the Alfvén radius of the host star ( $\sim 10$ stellar radii).

A detection of a magnetic field of a hot Jupiter would 1) provide a constraint on the rapid hydrodynamic escape of its atmosphere (Vidal-Madjar et al. 2003, 2004) which could affect the planet's structure and evolution, 2) present implications for the planet's internal structure, and 3) shed light on the mass-radius relationship of the known transiting planets (Pont et al. 2005, Bakos et al. 2006). Although the internal magnetic fields of hot Jupiters are expected to be weaker than Jupiter's due to probable tidal locking and slower spin rates (Sanchez-Lavega 2004, Griessmeier et al. 2004), Olson \& Christensen (2006) calculated that the magnetic field of a planet with even a tenth of Jupiter's rotation rate would still have a strong dipole moment, when reasonably assuming that the convection is not highly modified by the rotation rate. Also, the fact that both hot and very hot Jupiters, such as HD 209458 b and OGLE-TR-56 b, are detected at all means that they must have strong enough magnetic fields to balance the extreme stellar irradiation and CME plasma pressure to prevent destructive atmospheric erosion (Khodachenko et al. 2007).

\section{Measuring chromospheric activity}

Here, we compile échelle spectra collected between 2001 and 2006 of two hot Jupiter host stars, HD 179949 and $v$ And. The latest observations were made with the 3.6-m Canada-France-Hawaii Telescope (CFHT) on 7 nights in September 2005 and 9 nights in 2006 June. (Earlier observations are discussed in detail in Shkolnik et al. 2003 and 2005.) We used ESPaDOnS (Échelle SpectroPolarimetric Device for the Observation of Stars) to record the full spectrum over 40 grating orders covering 3700 to $10400 \AA$ at a spectral resolution $\mathrm{R}$ of $\approx 80,000$. The four nights of observations of $v$ And $(18,19,23,24$ September 2005) were taken in ESPaDOnS' 'spectropolarimetry' mode at R of 68,000. The reduction and analyses of these data are presented in Shkolnik et al. 2008.

The very strong Ca II H and K photospheric absorption lines suppress the local stellar continuum making it difficult to normalize each spectrum consistently. The normalization level was set at 0.3 of the flux at $3950 \AA$ centered on the $\mathrm{H}$ and $\mathrm{K}$ lines. Therefore the wavelengths were constant for all spectra of a given star, though they varied slightly from star to star due to variations in spectral type. The $\approx 7-\AA$ spectral range was chosen to isolate the $\mathrm{H}$ and $\mathrm{K}$ reversals. This window is wide enough that a few photospheric absorption features appear to test for general stability. To normalize each sub-spectrum, the end points were set to 1 and fitted with a straight line.

The spectra were grouped by date and a nightly mean was computed for each of the lines with non-varying $\mathrm{K}$ emission at the $\lesssim 0.001$ level on average on a given night. This is a result of $\mathrm{S} / \mathrm{N}$ variations and intranight (short time-scale) chromospheric activity.

We used nightly residuals from the average stellar spectrum to measure the chromospheric activity within the reversals. Each residual spectrum had a broad, low-order curvature removed. The residuals of the normalized spectra (smoothed by 17 pixels) were used to compute the mean absolute deviation $\left(\mathrm{MAD}=N^{-1} \Sigma \mid d a t a_{i}-\right.$ mean $\mid$ for $N$ spectra), a measure of overall variability within the span of the observing run.

\subsection{HD $179949 \& 6$ And: Evidence of the on/off nature of SPI}

When monitoring chromospheric emission, stellar activity may be modulated by the star's rotation, planetary motion in the case of SPI, or a combination of both. The orbital 


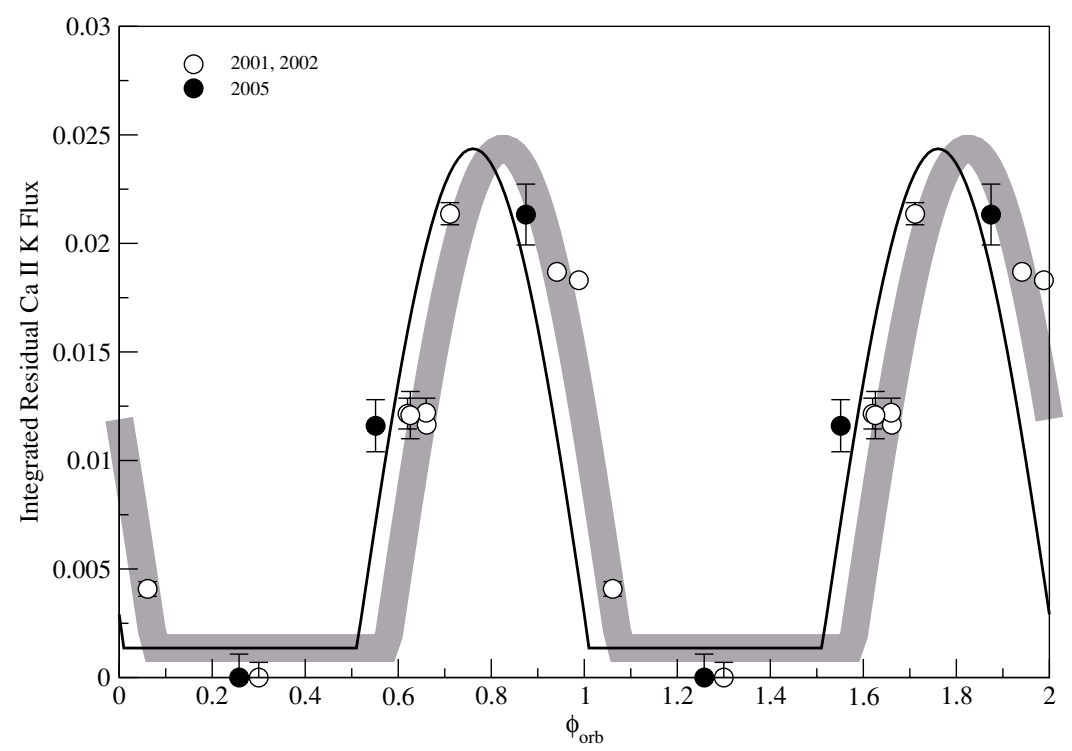

Figure 1. Integrated flux of the K-line residuals from a normalized mean spectrum of HD 179949 as a function of orbital phase for the 2001 and 2002 data (open circles) published in Shkolnik et al. (2003) and 2005 data (filled circles). The grey line is a best-fit spot model to the earlier data whose thickness reflects the error in the phase shift. The black line is the same fit slightly shifted in phase by -0.07 to better fit the 2005 data (See Shkolnik et al. 2008). This small shift relative to the earlier data is not signficant. Error bars in the integrated residual $\mathrm{K}$ flux are \pm the intranight residual RMS. Note that the data points are repeated for two cycles.

periods of the planets are well known and uniquely established by the PRV and transit discovery methods, but the rotation periods of the stars are much harder to determine in part due to stellar differential rotation. For studies of SPI, differentiating between rotational and orbital modulation of the chromospheric emission is key.

In Shkolnik et al. (2005) we presented evidence of planet-induced heating on HD 179949. The effect lasted for over a year and peaked only once per orbit, suggesting a magnetic interaction. In the simplest configuration, a magnetic interaction would occur near the sub-planetary point, when the planet is in front of the star relative to the line-of-sight, which defines orbital phase $\phi_{o r b}=0$. Reproduced in Figure 1, we fitted a truncated, best-fit spot model to our 2001 and 2002 data with $P=P_{\text {orb }}=3.092 \mathrm{~d}$ corresponding to the change in projected area of a bright spot on the stellar surface before being occulted by the stellar limb. The fit to the 2001 and 2002 data peaks at $\phi_{\text {orb }}=0.83 \pm 0.04$ with an amplitude of 0.027 . We over-plot new data from 2005 which is fit remarkably well by the same model with only an insignificantly small relative phase shift of -0.07 .

This phase lead may help identify the nature of the interaction. For example, the offset from the sub-planetary point of a starspot or group of starspots can be a characteristic effect of tidal friction, magnetic drag or reconnection with off-center stellar magnetic field lines. For further discussion on such mechanisms see papers by Gu et al. (2005), Preusse et al. (2006) and McIvor et al. (2006). In any case, the phasing, amplitude and period of the activity have persisted for over 4 years. 


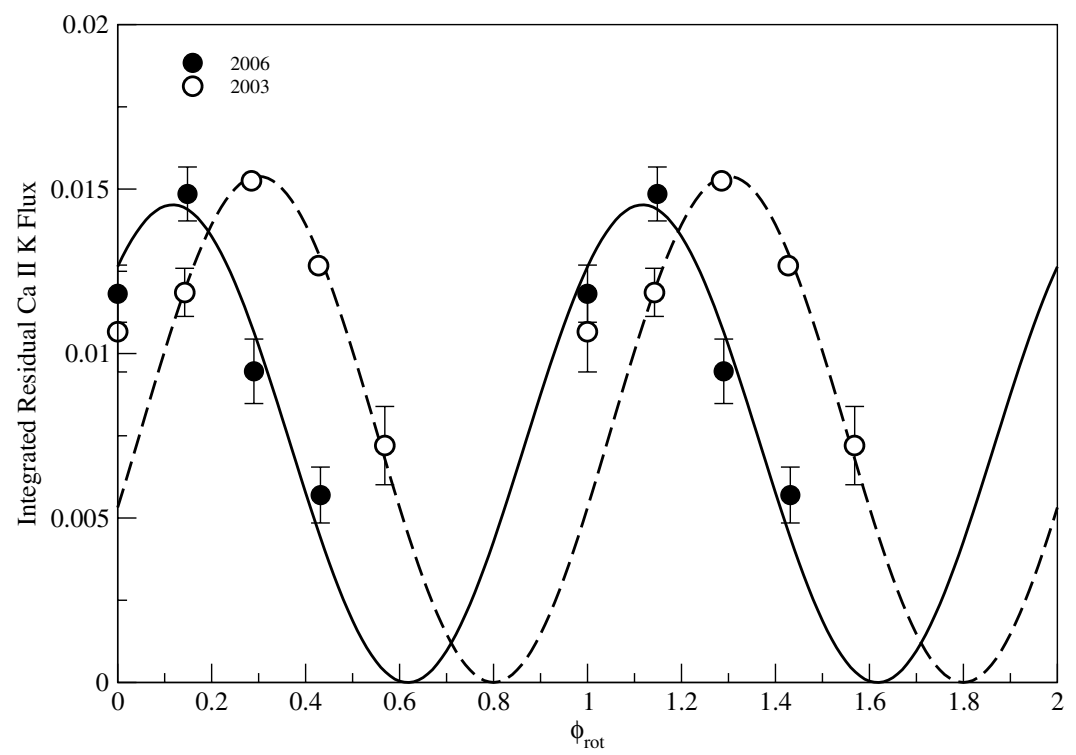

Figure 2. Integrated flux of the K-line residuals from a normalized mean spectrum of HD 179949 for 2003 and 2006 data plotted on a 7-day rotation period with phases relative to the first night of each run. The points are vertically shifted such that the minimum of each curves is zero. Error bars are \pm the intranight residual RMS. The curves are best-fit spot models to the two data sets.

Ca II data acquired in 2003 and 2006 of HD 179949 do not phase with the planet's orbit, but both phase well with a 7-day period, likely the rotation period of the star. In Figure 2 we fit data from each year separately with a rotation curve because the effects of differential rotation and the appearance and disappearance of new spots over the three years would produce variations in phase, amplitude and period in the observed modulation. Note that the amplitude of the rotational activity is only 0.6 of that of induced by SPI. Indirect indications of the rotation rate of HD 179949 imply $P_{\text {rot }} \approx 9$ days and are presented in Shkolnik et al. (2003) and Saar et al. (2004). Wolf \& Harmanec (2004) weakly detect $(1.5 \sigma)$ a photometric rotation period for HD 179949 of $7.07 \mathrm{~d}$ with an amplitude of only 0.008 mag. While more photometry is needed to determine a rotation period conclusively, the modulated Ca II emission of this star in both 2003 and 2006 strongly suggests a rotation period of 7 days.

Similarly, previous Ca II data of $v$ And indicated possible SPI (Figure 8 of Shkolnik et al. 2005), yet our September 2005 data appears to vary with the rotation. Again, the rotation period is not well known. Henry et al. (2000) quotes both 11 and 19 days, with a probable 11.6-day period from the $\left\langle S_{H K}\right\rangle$ index. Unlike data from 2002 and 2003, the 2005 data phase much better with $P_{r o t}=11.6 \mathrm{~d}$ than with a planetary orbit.

This on/off characteristic of SPI observed in the HD 179949 and $v$ And systems is explained by the models of Cranmer \& Saar (2007). They model the Ca II H \& K light curve of a sun-like star with a hot Jupiter interacting with the field geometry at various stages of the empirically derived solar magnetic field at annual steps of the 11year solar cycle. They conclude that due to the complex nature of the multipole fields, the Ca II K light curves due to SPI do not repeat exactly from orbit to orbit, and at 


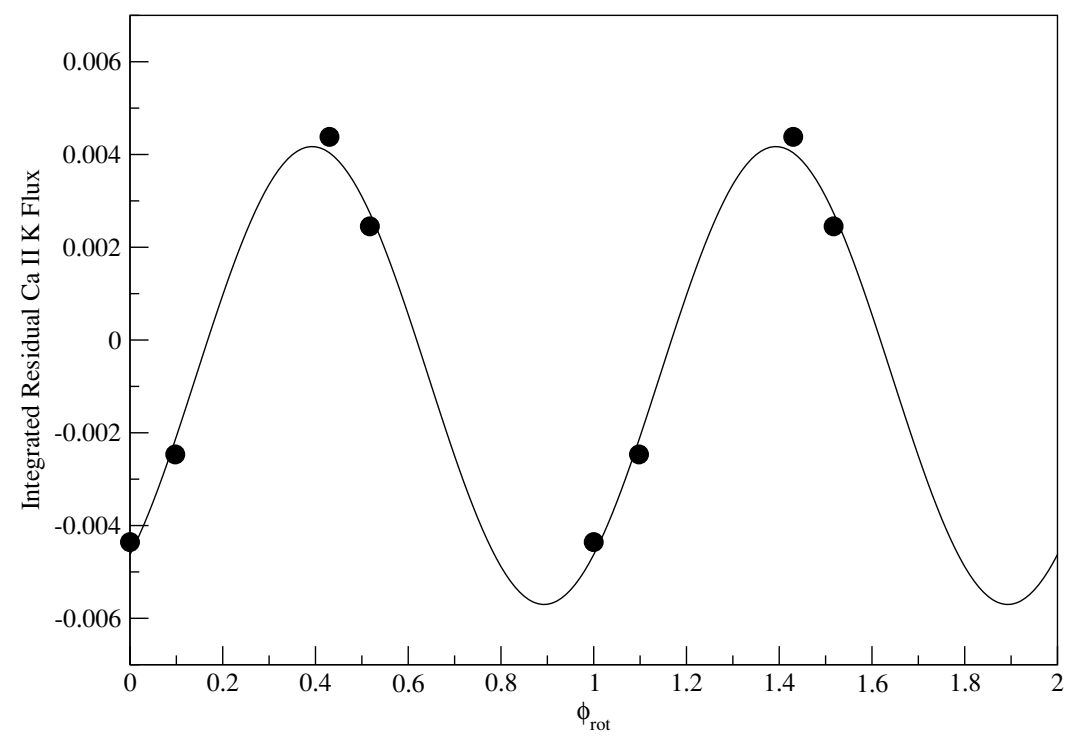

Figure 3. Integrated flux of the K-line residuals from a normalized mean spectrum of $v$ And for September 2005 data plotted on a 12-day rotation period. Error bars are within the size of the of the points. The curve is a best-fit spot model.

times the planet-induced enhancement may disappear altogether leaving only rotationally modulated emission. This may explain the 2003 and 2006 disappearance of the strong orbital modulation seen in 2001, 2002 and 2005 for HD 179949. Their models also show that for sparsely sampled data, the apparent phase shift between the peak Ca II emission and the sub-planetary point may fall between 0.2 and +0.2 (or $\pm 72^{\circ}$ ), consistent with the -0.17 phase shift we detected repeatedly for HD 179949.

\subsection{Night-to-night activity correlates with planet's magnetic moment}

Sánchez-Lavega (2004) looked at the internal structure and the convective motions of giant extrasolar planets in order to calculate their dynamo-generated surface magnetism. Given the same angular frequency (which is a reasonable approximation for the shortperiod planets in question), the magnetic dipole moment, and hence the magnetospheric strength, increases with planetary mass. This is observed for the magnetized planets in our own solar system, where the magnetic moment grows proportionally with the mass of the planet (Stevens 2005), and more specifically, with the planet's angular momentum $\left(L \propto M_{p} R_{p}^{2} P_{p, \text { rot }}^{-1}\right.$, Arge et al. 1995). Since only lower limits exist for the masses of most hot Jupiters and at such small semi-major axes they should be tidally locked $\left(P_{p, r o t}=\right.$ $\left.P_{\text {orb }}\right)$, we plot $M_{p} \sin i / \mathrm{P}_{\text {orb }}$ against $\langle\mathrm{MADK}\rangle$, the average of the integrated MAD of the $\mathrm{K}$ line residuals per observing run, in Figure 4 of our complete sample of 13 systems. (The targets with system parameters are listed in Tables 1 of Shkolnik et al. 2005 and 2008.) Of our sample, $\tau$ Boo has the most massive planet and yet falls well below the correlation. This is consistent with the proposed Alfvén wave model where the near zero relative motion due to the tidal locking of both the star and the planet $\left(P_{*, \text { rot }}=P_{p \text {, rot }}\right.$ $\left.=P_{\text {orb }}\right)$ produces minimal SPI because of the weak Alfvén waves generated as the planet 
passes through the stellar magnetosphere, thereby transporting little excess energy to the stellar surface along the magnetic field lines ( $\mathrm{Gu}$ et al. 2005). If this correlation between short-term activity and planetary magnetic moment holds for more hot Jupiter systems engaging in SPI, this could provide an empirical tool with which to estimate the strength of extrasolar planetary magnetic fields.

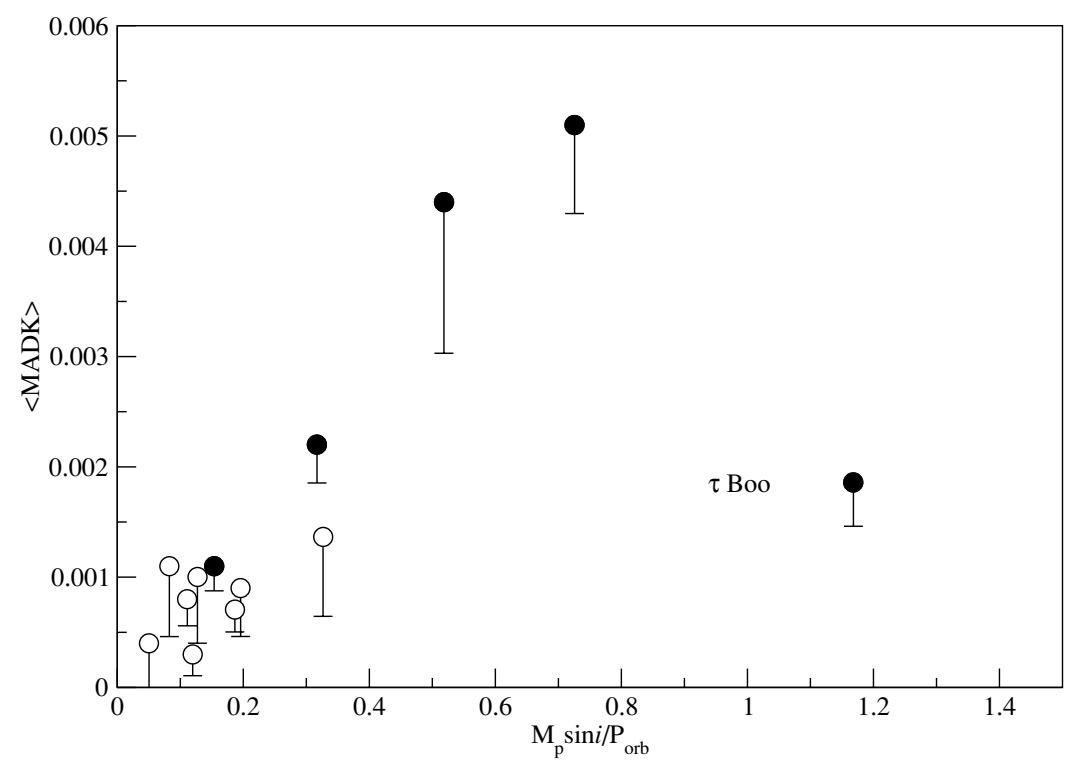

Figure 4. The ratio of the minimum planetary mass (in Jupiter masses) to the orbital period (in days) plotted against the average MAD of the K-line per observing run for all 13 stars observed. The x-axis quantifies the planet's magnetic moment assuming tidal locking, such that $P_{\text {rot }}=P_{\text {orb }}$. The filled-in circles are of stars which exhibit significant night-to-night variability in the Ca II K line: HD 73526, $v$ And, HD 179949 and HD 189733 (this work). The tidally-locked system of $\tau$ Boo does not follow the correlation traced out by the others. The error bars are one-sided due to the positive contribution of integrated MAD immediately outside the Ca II emission core and reflect the $\mathrm{S} / \mathrm{N}$ obtained for each target.

\section{Summary}

For our prime target, HD 179949, we now have a total of six observing runs spanning 5 years. During four runs (Aug 2001, July 2002, Aug 2002 and Sept 2005) the Ca II emission varied with the orbital period of 3.092 days, with consistent amplitude and peak phase indicative of a magnetic interaction between the star and planet. The peak activity on HD 179949 in these epochs occurs at $\phi_{\text {orb }} \approx 0.8$, leading the sub-planetary longitude by some $70^{\circ}$. The phase lead can provide information on the field geometries (i.e. Parker spiral) and the nature of the effect such as tidal friction, magnetic drag or reconnection with off-center magnetic fields.

HD 179949 data from the other two runs (Sept 2003 and June 2006) clearly vary with the rotation period of 7 days. A similar effect is seen on $v$ And where one of four epochs appears to be modulated by rotation rather than the planet's motion. This on/off behavior has been modeled by Cranmer \& Saar (2007) to be an effect of magnetic reconnection with the stellar field as it varies with the star's long-term activity cycle. 
To date we have observed 13 stars with hot Jupiters at CFHT and VLT, of which 5 appear to be actively engaging in SPI: HD 179949, $v$ And, HD 189733, HD 73256 and $\tau$ Boo. The activity as measured by the mean absolute deviation over a run on the first four of these stars correlates well with $M_{p} \sin i / P_{\text {orb }}$, a value proportional to the planet's magnetic moment, and thus with the hot Jupiter's magnetic field strength. Because of their small separation $(\leqslant 0.075 \mathrm{AU})$, a hot Jupiter lies within the Alfvén radius of its host star, allowing a direct magnetic interaction with the stellar surface. Although this correlation is tentative, short-term chromospheric variability may be our first probe of extrasolar planetary magnetospheres.

\section{Acknowledgements}

We are very grateful to John Barnes for contributing several $v$ And spectra, to JeanFrancois Donati for making Libre Esprit available to CFHT users, to the CFHT staff for their excellent support of this program, and to Benjamin Brown for useful discussions on planetary dynamos. Research funding from the NASA Postdoctoral Program (formerly the NRC Research Associateship) for E.S. and the National Research Council of Canada (D.A.B.) are gratefully acknowledged.

\section{References}

Arge, C. N., Mullan, D. J. \& Dolginov, A. Z., 1995, ApJ, 443, 795

Bakos, G. Á., Knutson, H., Pont, F., Moutou, C., Charbonneau, D., Shporer, A., Bouchy, F., Everett, M., Hergenrother, C., Latham, D. W., Mayor, M., Mazeh, T., Noyes, R. W., Queloz, D., Pál, A., \& Udry, S., 2006, ApJ, 650, 1160

Cranmer, S. \& Saar, S., 2007, arXiv:astro-ph/0702530v1

Cuntz, M., Saar, S.H., \& Musielak, Z.E., 2000, ApJ, 533, L151

Donati, J.-F., Semel, M., Carter, B. D., Rees, D. E., \& Collier Cameron, A. 1997, MNRAS, 291, 658

Donati, J.-F. 2007, MNRAS, in preparation

Griessmeier, J. -M. Stadelmann, A., Penz, T., Lammer, H., Selsis, F., Ribas, I., Guinan, E. F., Motschmann, U., Biernat, H. K., \& Weiss, W. W., 2004, A\&A, 425, 753

Gu, P.-G., Shkolnik, E., Li, S.-L., \& Liu, X-W, 2005, AN, 326, 909

Ip, W.-H., Kopp, A., \& Hu, J.-H., 2004, ApJ, 602, L53

Khodachenko, M. L., Lammer, H., Lichtenegger, H. I. M., Langmayr, D., Erkaev, N. V., Griemeier, J.-M., Leitner, M., Penz, T., Biernat, H. K., Motschmann, U., \& Rucker, H. O., 2007, P\&SS, 55, 631

McIvor, T., Jardine, M., \& Holzwarth, V., 2006, MNRAS, 367, 1

Olson, P. \& Christensen, U. R., 2006, E\&PSL. 250, 561

Pont, F., Bouchy, F., Melo, C., Santos, N. C., Mayor, M., Queloz, D., \& Udry, S., 2005, A\&A, 438,1123

Preusse, S., Kopp, A., Büchner, J., \& Motschmann, U. 2006, A\&A, 460, 317

Saar, S. H., Cuntz, M., \& Shkolnik, E., 2004, in IAU Symp. 219, Stars as Suns: Activity, Evolution, Planets, eds. A.K. Dupree and A.O. Benz, p.355

Sánchez-Lavega, A., 2004, ApJ, 609, 87

Shkolnik, E., Walker, G. A. H., \& Bohlender, D. A., 2003, ApJ, 597, 1092

Shkolnik, E., Walker, G. A. H., Bohlender, D. A., Gu, P. -G., \& Kürster, M., 2005, ApJ, 622, 1075

Shkolnik, E., Bohlender, D. A., Walker, G. A. H., \& Collier Cameron, A., 2008, ApJ, 676, 628

Stevens, I. R. 2005, MNRAS, 356, 1053

Vidal-Madjar, A., Lecavelier des Étangs, A., Désert, J.-M., Ballester, G., Ferlet, R., Hébrard, G., \& Mayor, M., 2003, Nature, 422, 143 
Vidal-Madjar, A., Désert, J.-M., Lecavelier des Etangs, A., Hébrard, G., Ballester, G. E., Ehrenreich, D., Ferlet, R., McConnell, J. C., Mayor, M., \& Parkinson, C. D., 2004, ApJ, 604, L69

Wolf, M. \& Harmanec, P., 2004, IBVS, 5575, 1 